\title{
Prognostic relevance of activated Akt kinase in node-negative breast cancer: a clinicopathological study of 99 cases
}

\author{
Klaus Jürgen Schmitz ${ }^{1}$, Friedrich Otterbach ${ }^{1}$, Rainer Callies ${ }^{2}$, Bodo Levkau ${ }^{3}$, Melanie \\ Hölscher $^{1}$, Oliver Hoffmann ${ }^{2}$, Florian Grabellus ${ }^{1}$, Rainer Kimmig ${ }^{2}$, Kurt Werner Schmid ${ }^{1, *}$ \\ and Hideo Andreas Baba ${ }^{1}$ \\ ${ }^{1}$ Institute of Pathology; ${ }^{2}$ Department of Obstetrics and Gynecology and ${ }^{3}$ Department of Patho-Physiology \\ University of Essen, Germany
}

\begin{abstract}
Patients with lymphnode-negative breast cancer show a 10 -year tumor recurrence rate of approximately $30 \%$. Therefore, it is important to identify high-risk patients who would benefit from further adjuvant therapy. For this purpose, we examined the activation state of two kinases important in the regulation of cell proliferation and apoptosis in a series of 99 node-negative breast cancer cases with a mean follow-up of 10 years: Akt and extracellular regulated kinase (ERK1/2). The activation of Akt and ERK1/2 was investigated by immunohistochemistry using phospho-specific antibodies. The results were correlated with HER-2/neu expression, histological grading, receptor status, overall survival (OS) as well as with cell proliferation (Ki67 immunoreactivity, mitotic count) and tumor apoptosis assessed by TUNEL staining. Activation of Akt (pAkt) but not activation of ERK1/2 (pERK1/2) correlated with HER-2/neu overexpression $(P<0.05)$ and was related to reduced tumor apoptosis $(P<0.05)$. No association was found between pAkt or $p E R K 1 / 2$ with cell proliferation assessed by Ki67 and mitotic count (MC). Survival analysis of receptor status, HER2/neu expression, histological grading, MC and pAkt immunoexpression showed a significant correlation with decreased OS, but only pAkt reached statistical significance in the multivariate Cox regression analysis $(P=0.015)$. Activation of Akt in node-negative breast cancer may indicate aggressive tumor behavior and may constitute an independent prognostic factor of OS. The determination of pAkt status may be of value in identifying high-risk patients, who would benefit from adjuvant therapy, and gives a rationale to investigate new therapy strategies by specific inhibition of the Akt signaling pathway in breast cancer.
\end{abstract}

Modern Pathology (2004) 17, 15-21, advance online publication, 19 November 2003; doi:10.1038/modpathol.3800002

Keywords: Akt; node-negative breast cancer; prognosis; HER-2/neu; ERK; MAP kinases; apoptosis

Breast cancer is a major cause of death among women, thus representing an important health problem. Adjuvant systemic therapy may considerably improve survival rates, but is on the other hand associated with toxic side effects. This conflict of interest is a problem particularly in patients with node-negative breast cancer, a group of patients with excellent prognosis and a 10-year recurrence rate of $30 \%$. Routine adjuvant therapy for node-negative breast cancer is thus difficult to justify. Therefore, the identification of novel markers for node-negative high-risk patients, who would benefit from adjuvant therapy, is of major importance.

Correspondence: HA Baba, Institute of Pathology, D-45122 Essen, Hufelandstr. 55, Germany.

E-mail: hideo.baba@medizin.uni-essen.de

* Member of the West German Cancer Centre (WTZE).

Received 22 March 2003; revised 20 August 2003; accepted 16 September 2003; published online 19 November 2003
Signal transduction and modulation represent central mechanisms in cellular processes such as cell cycle regulation, oncogenesis and apoptosis. As tumor progression is based on a disturbed balance between proliferation and apoptosis, we investigated two kinases that are involved in these important cellular events: Akt and ERK1/2.

The Akt signaling pathway plays a central role in tumorigenesis. ${ }^{1}$ Akt is a serine/threonine kinase (also named protein kinase $\mathrm{B}$ [PKB]) and its activation is induced by phosphorylation mediated by PI3K in association with tyrosine kinase receptors. PI3K is localized upstream of the Akt kinase and is essential for the activation of Akt. The Akt kinase promotes cell survival by inhibition of apoptosis via phosphorylation of proapoptotic proteins such as Bad, forkhead transcription factor and caspase 9. ${ }^{2,3}$ However, in addition to its antiapoptotic function, Akt is involved in cell proliferation by 
regulation of the cyclin-dependent kinase inhibitors. $^{4,5}$

Among the tumor-cell survival pathways, those mediated by the Akt kinase are the most critical. ${ }^{6,7}$ Moreover, Akt transfection results in a malignant phenotype as determined by growth in soft agar and tumor formation in nude mice. ${ }^{8}$ In human malignancies, Akt is activated in a variety of carcinomas including prostate, ovary and breast cancer. ${ }^{8,9}$ In a recent study including both node-positive and negative breast cancer patients, activation of Akt was seen in $54 \%$ of 93 cases after endocrine therapy. Survival analysis revealed that patients with pAktpositive tumors were more prone to relapse with distant metastasis. ${ }^{10}$

The ERK signaling pathway represents a component of the mitogen activated protein kinases (MAPK) cascade and is activated by extracellular, frequently mitogenic ligands resulting in increased cellular proliferation in vivo. ${ }^{11,12}$ In human breast cancer, activated ERK1/2 kinases were associated with lower survival rates, suggesting a prognostic value in primary breast cancer patients. ${ }^{13,14}$

Poor prognosis and resistance to systemic therapy were found in breast cancer patients overexpressing the tyrosine kinase receptor encoded by the oncogene HER-2/neu. Studies on breast cancer cell lines have shown a crosstalk between HER-2/neu overexpression and activation of the Akt signaling pathway. ${ }^{15,16}$ Akt activation was observed in HER-2/ neu-positive breast cancer and may be responsible for higher tumor aggressiveness by increased resistance to stress-induced apoptosis. ${ }^{17}$

In this study, we examined the activation of Akt and ERK1/2 in node-negative breast cancer. We found Akt but not ERK1/2 phosphorylation to be an independent prognostic factor related to HER-2/neu overexpression. We provide evidence that Akt activation is associated with decreased tumor apoptosis, which may at least partly explain the poorer prognostic outcome.

\section{Materials and methods}

\section{Patients}

This study comprised 106 female breast cancer patients (mean age 55 years) from the Department of Gynecology (University of Essen, Germany), who underwent surgery between 1989 and 1993. Complete clinical records and follow-up information were available in all cases. The negative lymph node status was confirmed by axillary dissection. All surgical material was fixed in $4 \%$ formalin and routinely processed. The tumors were classified according to the pTNM System (5th edition) and graded according to Elston and Ellis. ${ }^{18}$ Out of 106 patients, 27 died during follow-up, and in one case the cause of death was unknown. In all, 19 patients died of breast cancer, and seven were excluded from this study, having died from either
Table 1 Clinicopathological data (99 node-negative breast cancer)

\begin{tabular}{lcc}
\hline Parameter & Number & $\%$ \\
\hline Tumor type & & \\
Invasive ductal and mixed & 71 & 71.7 \\
Invasive lobular & 17 & 17.2 \\
Others & 11 & 11.1 \\
Tumor size & & \\
pT1(a,b,c) & 61 & 61.6 \\
pT2 & 35 & 35.4 \\
pT3 and 4 & 3 & 3 \\
& & \\
Histological grading & 18 & 18.2 \\
Well & 59 & 59.6 \\
Moderate & 22 & 22.2 \\
Poor & & \\
& & \\
ER (PR) status & & $70.3(38.9)$ \\
Positive & & 29.9 \\
Negative & & \\
HercepTest & & \\
Negative $(0,1+)$ & 68 & \\
Positive (2+, 3+) & 29 & \\
\hline
\end{tabular}

Data available for HercepTest in 97 cases.

benign or other cancer diseases. Statistical analysis of the remaining 99 cases was based on a mean follow-up period of 10 (mean 9.9) years. In 51 cases, mastectomy had been performed (51.5\%), and 48 patients were treated with local excisions $(48.5 \%)$. In total, 74 patients were treated by surgery alone, whereas 21 patients received adjuvant radiation and four underwent adjuvant endocrine therapy. Table 1 summarizes the clinicopathological parameters of this study.

\section{Phospho-Akt and ERK1/2 Immunostaining}

Human Akt exists as three isoforms: Akt1/PKB $\alpha$, $\mathrm{Akt} 2 / \mathrm{PKB} \beta$ and $\mathrm{Akt} 3 / \mathrm{PKB} \gamma$. In the present study, we used a nonisoform specific antibody reacting with Akt1 when phosphorylated at serine 473 and Akt2/3 when phosphorylated at sites equivalent to the serine 473 site of Akt1. ${ }^{19}$ Rabbit polyclonal phospho-Akt antibody (Ser473; Cell Signaling Technology, Beverly, MA, USA) was used at 1:200 dilution, and monoclonal phospho-p44/42 MAP kinase antibody (Thr 202/Tyr 204; Cell Signaling Technology) at 1:100 dilution. Immunocytochemistry was performed on $5 \mu \mathrm{m}$ thick paraffin sections. Antigen retrieval was carried out with $0.01 \mathrm{M}$ citrate buffer at $\mathrm{pH} 6.1$ for $20 \mathrm{~min}$ (phospho-Ser $473 \mathrm{PKB} / \mathrm{Akt}$ ), respectively, $40 \mathrm{~min}$ (phospho ERK1/2) in a hot water bath $\left(95^{\circ} \mathrm{C}\right)$. Both antibodies were incubated overnight in a humidified chamber at $4^{\circ} \mathrm{C}$. The APAAP method was used for antibody demonstration. Paraffin slides of a human prostate cancer cell line (LNCaP), treated and untreated with an inhibitor of PI3Kinase (LY294002), served as Akt- 
positive and -negative control. Untreated LNCap cells revealed a strong membranous immunoreactivity with the pAkt antibody whereas treated LNCap cells failed to show an immunoreaction.

\section{Semiquantification of Akt and ERK1/2 Staining}

Tumor cells with easily detectable specific immunostaining, independent of the amount of stained cells, were scored as strongly positive $(2+)$. Tumors exhibiting a detectable but faint immunostaining were scored as weak $(1+)$, whereas tumors with a minimal, hardly detectable or missing staining pattern were classified as negative (0).

\section{Receptor Status and HER2 Protein Expression}

The estrogen (ER) and progesterone receptor (PR) status of the tumors was determined using monoclonal anti-human antibodies (ER: clone 1D5, Code No. M 7047; PR: clone PgR 636, Code No. M 3569; DAKO). After antigen retrieval the primary antibodies were incubated for $30 \mathrm{~min}$ (PR; dilution: $1: 50$ ), respectively, $2 \mathrm{~h}$ (ER; dilution $1: 25)$. The monoclonal PowerVision (No. DPVM-110AP, ImmunoVision Tech. Co) served as a detection system. Semiquantitative evaluation followed. A tumor was regarded as receptor negative if none or less than $10 \%$ of the tumor cells showed weak or missing nuclear immunostaining. For statistical analysis, cases were subdivided into a negative and a positive group. The DAKO HercepTest ${ }^{\mathrm{TM}}$ was used to detect the HER2/neu protein expression (DAKO, No. K 5204). Staining procedures were performed following the manufacturer's protocols. For statistical analysis, negative (DAKO score 0 and $1+$ ) and positive (DAKO score $2+$ and $3+$ ) groups were created.

\section{Ki67 Immunostaining, TUNEL}

The Ki67 antigen was detected using a standard APAAP method. After antigen retrieval, the prediluted monoclonal anti-Ki67 antibody (Biogenex, San Ramon, USA) was incubated for $2 \mathrm{~h}$ in a humidified chamber. The growth fraction (GF) was defined as the percentage of Ki67-positive nuclei per 500 tumor cells. In situ DNA fragmentation was established using the terminal desoxyribonucleotide transferase (TdT)-mediated dUTP nick end labeling technique (TUNEL) in paraffin-embedded sections. We used the ApoTag ${ }^{\mathrm{TM}}$ Plus Peroxidase in Situ Apoptosis Detection Kit (Intergen Company). Staining procedures were again performed following the manufacturer's recommendations. Incubation with $20 \mu \mathrm{g} / \mathrm{ml}$ proteinase $\mathrm{K}$ solution was modified to $10 \mathrm{~min}$ in order to achieve maximum staining results. Apoptotic count was performed using a light microscope, counting stained apoptotic tumor cells per 10 high-power fields $(\mathrm{HPF})(\times 400$ magnification). Corresponding $\mathrm{H} \& \mathrm{E}$ sections were analyzed to avoid miscounting necrotic cells. In 10 cases, the amount of tumor tissue available was not sufficient for adequate counting.

\section{Mitotic Count (MC)}

MC was carried out taking into consideration the actual area of $10 \mathrm{HPF}$ of the Leica DMLB microscope. MC results were grouped as 0-10, 11-21, and more than 21 per HPF.

\section{Statistical Analysis}

Immunostainings were assessed independently by two of the authors (KJS and FO) in a blind-trial fashion without a knowledge of the clinical outcome. In case of disagreement, slides were reevaluated by both and a final decision was made. There was a very high interobserver concordance of pAkt staining scores (Spearman rank-correlation coefficient: $r=0.904 ; P<0.001)$ and the staining results were highly reproducible $(r=0.710$; $P<0.001)$. All data were converted to a PC and statistically analyzed using SPSS Version 10 for Windows. Relationships between ordinal parameters were investigated using two-tailed $\chi^{2}$ analysis (or Fisher's exact test where patient numbers were small). The relationship between apoptotic count and pAkt was determined using the MannWhitney's U test. Overall survival (OS) curves were estimated using the Kaplan-Meier method, and any differences in the survival curves were compared by the log-rank test. For multivariate analysis, the Cox regression was used. For both tests, a $P$-value of 0.05 or less was considered to be of statistical significance. Overall, 95\% confidence intervals (95\% CI) were used throughout.

\section{Results}

\section{Phospho-Akt Immunostaining}

Prominent cytoplasmic and partly nuclear pAkt immunoreactivity was limited to tumor cells, whereas non-neoplastic breast tissue revealed minimal or no staining. Occasionally, staining heterogeneity was apparent at the invasive tumor front. Of all the patients examined, 35 cases (35.4\%) were classified as negative for phospho-Akt, 57 (57.6\%) breast cancers showed a weak mainly cytoplasmatic immunostaining and seven $(7.1 \%)$ cases exhibited a strong cytoplasmatic as well as nuclear immunostaining. Five out of these seven tumors in this group were moderately differentiated (71.4\%), while two of the seven tumors were poorly differentiated. Among the pAkt-overexpressing subgroup, three patients underwent mastectomy and four were treated by local excision; two received adjuvant 
therapy. No correlation was found between pAkt and tumor size, GF, MC, grading or receptor status.

\section{Phospho-ERK1/2 Immunostaining}

Tumor cells exhibited strongest nuclear and cytoplasmatic immunostaining while non-neoplastic breast tissue only occasionally revealed a weak staining. Similar to pAkt, heterogenous immunostaining for $\mathrm{pERK} 1 / 2$ was detected at the invasive tumor front. In all, $66 \quad(66.7 \%)$ tumors were classified as negative, 25 tumors $(25.3 \%)$ exhibited a weak staining, eight $(8.1 \%)$ tumors showed a strong cytoplasmatic and nuclear staining. Statistical analysis failed to show any relationship between pERK1/2 and pAkt, histological grading, GF, MC, tumor size, TUNEL, HER-2/neu overexpression, receptor status or OS (data not shown).

\section{HER-2/neu Status}

A total of 97 tumors were analyzed for HER-2/neu protein expression: $26(26.9 \%)$. were classified as negative (0), $42(43.3 \%)$ as negative $(1+), 15$ $(15.5 \%)$ as weakly positive $(2+)$ and $14(14.4 \%)$ as strongly positive $(3+)$. Tumors with a score $2+$ and $3+$ were defined as HER-2/neu positive. Five of the seven $(71.4 \%)$ tumors classified as strongly pAkt positive showed HER-2/neu protein overexpression, while pAkt-negative or -weakly positive tumors exhibited in less than 30\% a HER-2/neu overexpression. Statistical analysis revealed a significant relationship of HER-2/neu overexpression $(2+$ and $3+)$ with pAkt-immunostaining intensity $(P=0.045$, Figure 1).

\section{Lower Apoptotic Rate in pAkt-immunopositive Tumors}

The number of apoptotic cells correlated directly with the GF determined by Ki67 immunostaining (Pearson's $r=0.427, P<0.01)$ and MC $(r=0.342$, $P<0.01)$. There was a significantly lower number of apoptotic cells per $10 \mathrm{HPF}$ in pAkt weak/strong cases compared to pAkt-negative cases $(P<0.05$; Figure 2). All tumors, stratified according to their pAkt immunostaining intensity, revealed similar GFs and MC (data not shown). The number of apoptotic cells correlated with the histological grading. Poorly differentiated tumors exhibited significantly higher apoptotic cell counts per 10 HPF than moderate or well-differentiated tumors (data not shown). No relationship was seen between apoptosis and receptor status, respectively, HER-2/ neu status Representative immuno-histochemical stainings are shown in Figure 3.

\section{Prognosis}

Only two out of $35(5.7 \%)$ patients classified as pAkt negative in our study died of breast cancer. In contrast three out of seven $(42.8 \%)$ patients classi-

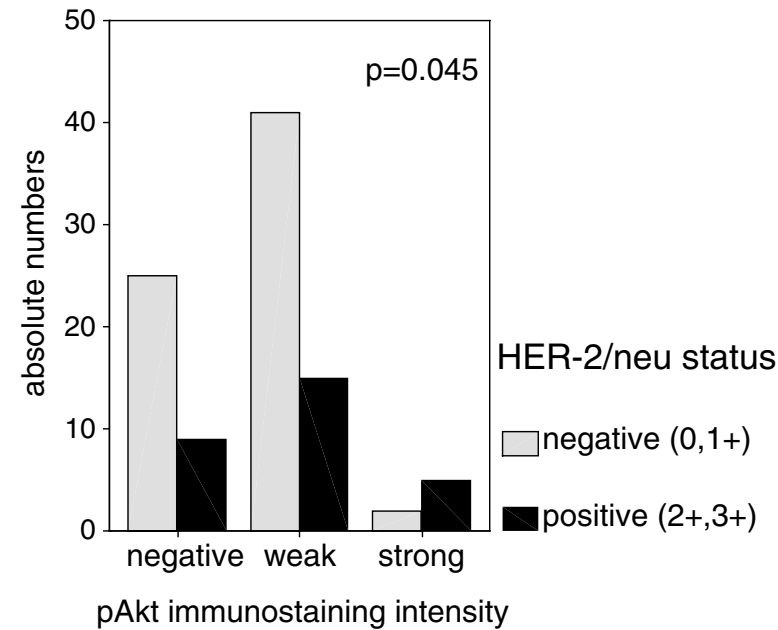

Figure 1 Number of HER-2/neu-overexpressing tumors $(2+$ and $3+)$ corresponding to pAkt immunoexpression. Tumors with strong Akt activation show significant HER-2/neu overexpression $\left(P=0.045 ; \chi^{2}\right.$ analysis $)$.

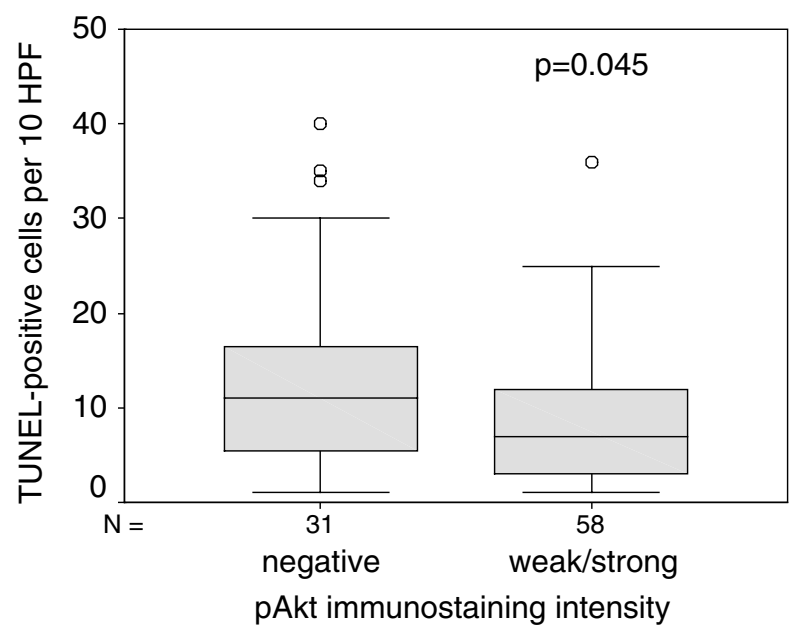

Figure 2 Boxplots showing the relation of pAkt immunostaining intensity and apoptosis detected by TUNEL staining. Tumors with weak/strong Akt phosphorylation are significantly associated with a reduced amount of TUNEL-positive cells $(P=0.045$; Mann-Whitney U test). Open circles indicate outliers. Data were available for 89 cases.

fied as strongly pAkt positive died from the same cause. In all, 14 of $57(24.6 \%)$ patients classified as weakly pAkt positive died of breast cancer; in one case, cause of death was unknown.

No significant difference in survival was detected between patients with diverse adjuvant or surgical treatment (data not shown). OS was inversely associated with different pAkt staining intensity (Figure 4; $P=0.0099$ ). Parallel analysis revealed that histological grading, ER status, HER-2/neu status and MC $(P<0.05)$ were significantly related to shorter OS (Table 2). The parameters tumor size $(P=0.08$; cutoff $1.5 \mathrm{~cm})$ and progesterone receptor status $(P=0.0612)$ tended to be associated with decreased OS but failed to reach statistical significance. 

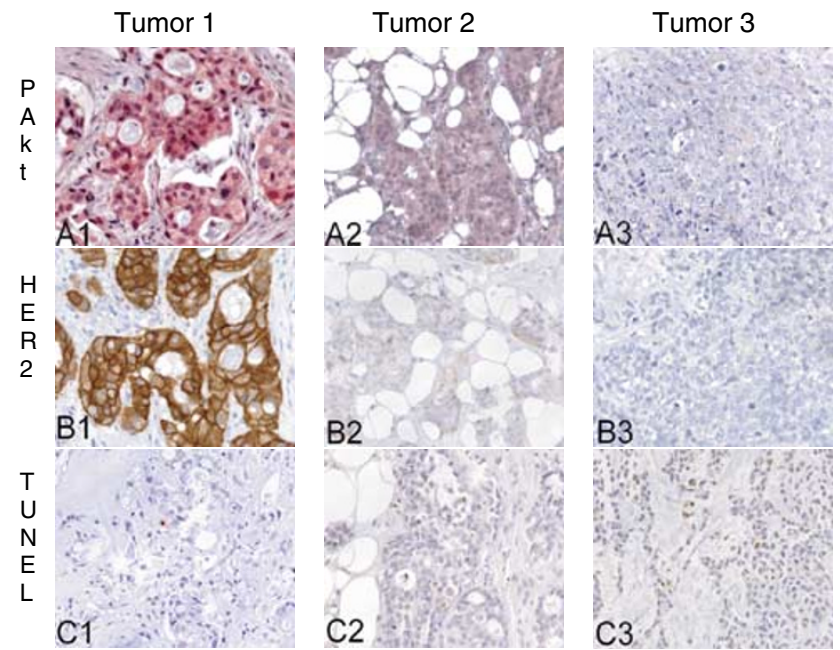

Figure 3 Representative immunohistochemical stainings of serial sections of three tumors for pAkt, HercepTest ${ }^{\mathrm{TM}}$ and TUNEL. A1A3: pAkt: The staining intensity of invasive carcinomas ranged from strong (A1) to weak (A2) and negative (A3). B1-B3: HercepTest ${ }^{\mathrm{TM}}$ : Tumors showing a strong pAkt immunostaining (A1) exhibited more frequently HER-2/neu protein overexpression (DAKO-Score $3+$ ) (B1) whereas tumors expressing a weak or none pAkt immunostaining were more often classified as DAKOScore $1+$ or 0 (B2; B3). C1-C3: TUNEL staining: Tumors with a strong pAkt immunostaining feature a decreased amount of TUNEL-positive cells (C1), weak or negative pAkt immunostaining is correlated with higher numbers of TUNEL-positive cells (C2-C3).

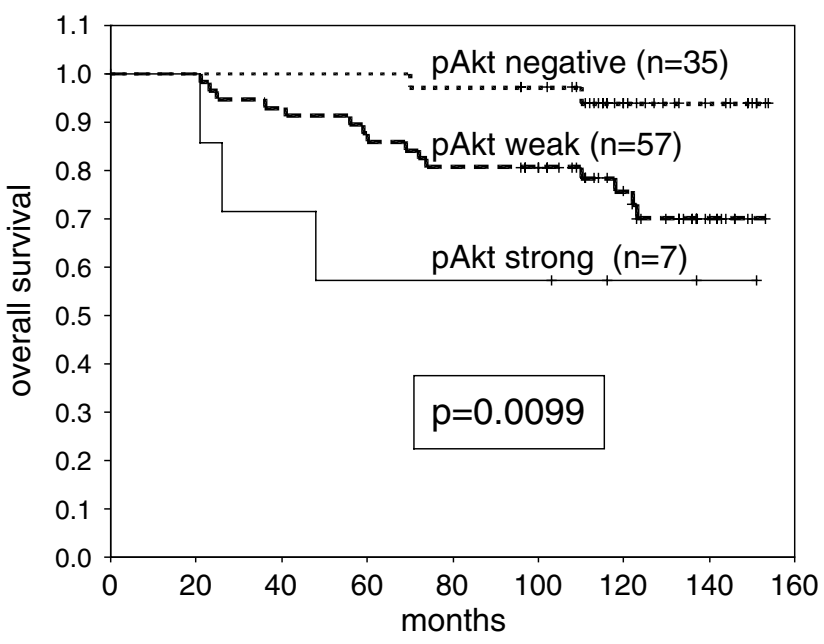

Figure 4 Kaplan-Meier survival plot of 99 node-negative breast cancers in relation to pAkt immunostaining intensity. Log-rank test: $P=0.0099$.

No significant relationship was observed between pERK1/2, Ki-67, apoptotic count and OS. OS and the relevant clinicopathological parameters (pAkt, histological grading, ER, MC and HER-2/neu) were subject to multivariate analyses. As a result, only pAkt was statistically associated with OS (log-rank test, $P=0.015$ ) in the group of node-negative breast cancer patients (Table 3 ).
Table 2 Univariate analysis (Kaplan-Meier, log-rank test) for prognostic significance of pAkt, histological grading, receptor status, HER-2/neu, MC, Ki-67 and tumor size

\begin{tabular}{lc}
\hline Parameter & P-value \\
\hline pAkt (negative/weak/strong expression) & $0.0099^{*}$ \\
Histological grade & $0.0072^{*}$ \\
ER status & $0.0150^{*}$ \\
HER2 status (HercepTest ${ }^{\mathrm{TM}}$ ) & $0.0487^{*}$ \\
MC $(0-10,11-21,>21)$ & $0.0182^{*}$ \\
Ki-67 $(<10 \%$ or $\geq 10 \%)$ & 0.1419 \\
Tumor size & 0.0801 \\
PR status & 0.0612
\end{tabular}

${ }^{*}$ Statistically significant.

Table 3 Results of multivariate analysis (Cox regression) to determine the independent prognostic value of different variables in relation to OS

\begin{tabular}{lccc}
\hline Covariate & Relative risk $\left(\mathrm{e}^{\beta}\right)$ & 95\% CI & P-value \\
\hline pAkt & 2.501 & $(1.194-5.239)$ & 0.015 \\
Histological grade $(1,2,3)$ & NS & NS & NS \\
$\begin{array}{l}\text { ER status } \\
\text { Negative vs positive }\end{array}$ & NS & NS & NS \\
$\begin{array}{l}\text { HER-2/neu } \\
\text { Positive vs negative }\end{array}$ & NS & NS & NS \\
$\begin{array}{l}\text { MC } \\
0-10 \text { vs } 11-21 \text { vs }>22\end{array}$ & NS & NS & NS \\
\hline
\end{tabular}

NS, statistically not significant.

\section{Discussion}

This study examined the activation of Akt and ERK1/2 in node-negative breast cancer by immunohistochemistry. We demonstrated that active Akt (pAkt) but not active ERK1/2 (pERK1/2) was significantly associated with a decreased OS in this collective of node-negative breast cancer patients and provide evidence that pAkt is related to decreased tumor apoptosis and HER-2/neu overexpression.

Activation of Akt results in pleiotrophic effects contributing to tumor aggressiveness. At least $13 \mathrm{Akt}$ substrates have been identified so far in mammalian cells. They can be categorized into two main classes: regulators of apoptosis on the one hand, and of cell growth (including protein synthesis and glycogen metabolism $)^{20}$ and cell cycle regulation ${ }^{4,5}$ on the other.

To determine which function of Akt may be associated with the shortened OS of patients with Akt-positive tumors, we focused on apoptosis (TUNEL) and cell proliferation (Ki67, Mc). Our data indicate that tumors with activated Akt exhibit a 
lower amount of TUNEL-positive cells compared to pAkt-negative tumors, implying a lower apoptotic rate. Although proliferation and apoptosis are assumed to play a key role in tumor progression, the clinical relevance of apoptotic frequency in breast cancer remains unclear. ${ }^{21}$ Several studies conducted on the possible prognostic value of apoptotic markers revealed varying results. While some studies report high levels of apoptosis to be associated with worse survival, ${ }^{22-24}$ the majority fail to demonstrate an independent prognostic value. ${ }^{25-28}$ In contrast, Tanaka et $a l^{29}$ demonstrated that loss of apoptosis due to the expression of an apoptosis inhibitor (survivin) was an independent prognostic parameter in breast cancer. Tumor growth is not only determined by tumor cell proliferation, but rather by the net result of proliferation and cell death. We did not find an increase in proliferative activity measured by Ki-67 immunoreactivity and MCs in cases of breast cancer with Akt activation; this suggests an impaired balance between cell loss and cell gain, resulting in a shift toward tumor net growth due to decreased apoptosis.

Contrary to a previous report ${ }^{13}$ describing a potential prognostic value of ERK1/2 MAP kinase activity in breast cancer independently of nodal status, we could not confirm this observation. This discrepancy may be explained by the patient selection in our cohort, which was focused on node-negative patients. Recent investigations revealed Akt as a downstream target of HER-2/neu signaling. ${ }^{17,30,31}$ The biological effects of HER-2/neu overexpression are mediated via several intracellular pathways regulating important downstream substrates including MAP kinase, phosphoinositide 3-kinase (PI3K) and $\mathrm{Akt}^{32}$ Dimerization of HER-2/ HER-3 has been shown to be connected to PI3K with subsequent phosphorylation of Akt. ${ }^{33}$ We report a significant relationship between pAkt and HER-2/ neu in our study, which may result from activation of Akt via HER-2/overexpression.

In a recent study, Perez-Tenorio and $\mathrm{Stal}^{10}$ advocated a prognostic relevance for Akt activation in human breast cancer but did not separate nodepositive and -negative patients. The findings presented here extend these observations by showing that Akt activation is an independent prognostic parameter in node-negative breast cancer. Since we have shown that activation of Akt coincides with reduced OS, treatment of certain patients with PI3K inhibitors may disclose new therapeutic options. In vitro, inhibitors of the PI3K signaling pathway such as Wortmannin and LY294002 have a proapoptotic effect. $^{34,35}$

In conclusion, our results suggest that activation of Akt in node-negative breast cancer indicates a more aggressive tumor behavior. The detection of Akt phosphorylation may be considered as a useful marker to identify high-risk patients, who would benefit from adjuvant therapy. However, due to the relatively small number of patients who died from breast cancer $(n=19)$ in this cohort $(n=99)$, these promising results need to be confirmed in larger cohorts.

The understanding of the Akt signaling pathway and connections to other signal transduction cascades may lead to new concepts for therapeutic strategies in human breast cancer. ${ }^{36}$

\section{Acknowledgement}

The technical assistance of Dorothe Möllmann and Annett Keller is gratefully acknowledged. This work was supported by the local research fund (IFORES) of the University of Essen. No financial relationship exists that might lead to conflict concerning the content of this study.

\section{References}

1 Testa JR, Bellacosa A. AKT plays a central role in tumorigenesis. Proc Natl Acad Sci USA 2001;98: 10983-10985.

2 Datta SR, Brunet A, Greenberg ME. Cellular survival: a play in three Akts. Genes Dev 1999;13: 2905-2927.

3 Cardone MH, Roy N, Stennicke HR, et al. Regulation of cell death protease caspase-9 by phosphorylation. Science 1998;282:1318-1321.

4 Zhou BP, Liao Y, Xia W, et al. Cytoplasmic localization of p21Cip1/WAF1 by Akt-induced phosphorylation in HER-2/neu-overexpressing cells. Nat Cell Biol 2001;3:245-252.

5 Shin I, Yakes FM, Rojo F, et al. PKB/Akt mediates cellcycle progression by phosphorylation of p27(Kip1) at threonine 157 and modulation of its cellular localization. Nat Med 2002;8:1145-1152.

6 Burgering BM, Coffer PJ. Protein kinase B (c-Akt) in phosphatidylinositol-3-OH kinase signal transduction. Nature 1995;376:599-602.

7 Chan TO, Rittenhouse SE, Tsichlis PN. AKT/PKB and other D3 phosphoinositide-regulated kinases: kinase activation by phosphoinositide-dependent phosphorylation. Annu Rev Biochem 1999;68:965-1014.

8 Sun M, Wang G, Paciga JE, et al. AKT1/PKBalpha kinase is frequently elevated in human cancers and its constitutive activation is required for oncogenic transformation in NIH3T3 cells. Am J Pathol 2001;159:431-437.

9 Bellacosa A, de Feo D, Godwin AK, et al. Molecular alterations of the AKT2 oncogene in ovarian and breast carcinomas. Int J Cancer 1995;64:280-285.

10 Perez-Tenorio G, Stal O. Activation of $\mathrm{AKT} / \mathrm{PKB}$ in breast cancer predicts a worse outcome among endocrine treated patients. Br J Cancer 2002;86:540-545.

11 Robinson MJ, Cobb MH. Mitogen-activated protein kinase pathways. Curr Opin Cell Biol 1997;9: 180-186.

12 Pearson G, Robinson F, Beers GT, et al. Mitogenactivated protein (MAP) kinase pathways: regulation and physiological functions. Endocr Rev 2001;22: 153-183.

13 Mueller H, Flury N, Eppenberger-Castori S, et al. Potential prognostic value of mitogen-activated protein kinase activity for disease-free survival of primary breast cancer patients. Int J Cancer 2000;89:384-388. 
14 Sivaraman VS, Wang H, Nuovo GJ, et al. Hyperexpression of mitogen-activated protein kinase in human breast cancer. J Clin Invest 1997;99:1478-1483.

15 Muthuswamy SK, Gilman M, Brugge JS. Controlled dimerization of ErbB receptors provides evidence for differential signaling by homo- and heterodimers. Mol Cell Biol 1999;19:6845-6857.

16 Ahmad S, Singh N, Glazer RI. Role of AKT1 in 17betaestradiol- and insulin-like growth factor I (IGF-I)dependent proliferation and prevention of apoptosis in MCF-7 breast carcinoma cells. Biochem Pharmacol 1999;58:425-430.

17 Bacus SS, Altomare DA, Lyass L, et al. AKT2 is frequently upregulated in HER-2/neu-positive breast cancers and may contribute to tumor aggressiveness by enhancing cell survival. Oncogene 2002; 21:3532-3540.

18 Elston CW, Ellis IO. Pathological prognostic factors in breast cancer I. The value of histological grade in breast cancer: experience from a large study with long term follow up. Histopathology 1991;19:403-410.

19 Vanhaesebroeck B, Alessi DR. The PI3K-PDK1 connection: more than just a road to PKB. Biochem J 2000;346(Part 3):561-576.

20 Blume-Jensen P, Hunter T. Oncogenic kinase signalling. Nature 2001;411:355-365.

21 Jager JJ, Jansen RL, Arends JW. Clinical relevance of apoptotic markers in breast cancer not yet clear. Apoptosis 2002;7:361-365.

22 de Jong JS, van Diest PJ, Baak JP. Number of apoptotic cells as a prognostic marker in invasive breast cancer. Br J Cancer 2000;82:368-373.

23 Gonzalez-Campora R, Galera Ruiz MR, Vazquez RF, et al. Apoptosis in breast carcinoma. Pathol Res Pract 2000;196:167-174.

24 Vakkala M, Lahteenmaki K, Raunio H, et al. Apoptosis during breast carcinoma progression. Clin Cancer Res 1999;5:319-324.

25 Lipponen P, Aaltomaa S, Kosma VM, et al. Apoptosis in breast cancer is related to histopathological characteristics and prognosis. Eur J Cancer 1994;30A:2068-2073.

26 Zhang GJ, Kimijima I, Abe R, et al. Apoptotic index correlates to bcl-2 and p53 protein expression, histological grade and prognosis in invasive breast cancers. Anticancer Res 1998;18:1989-1998.

27 Berardo MD, Elledge RM, de Moor C, et al, Osborne CK, Allred DC. bcl-2 and apoptosis in lymph node positive breast carcinoma. Cancer 1998;82: 1296-1302.

28 Liu S, Edgerton SM, Moore DH, et al. Measures of cell turnover (proliferation and apoptosis) and their association with survival in breast cancer. Clin Cancer Res 2001;7:1716-1723

29 Tanaka K, Iwamoto S, Gon G, et al. Expression of survivin and its relationship to loss of apoptosis in breast carcinomas. Clin Cancer Res 2000;6:127-134.

30 Altiok S, Batt D, Altiok N, et al. Heregulin induces phosphorylation of BRCA1 through phosphatidylinositol 3-Kinase/AKT in breast cancer cells. J Biol Chem 1999;274:32274-32278.

31 Liu W, Li J, Roth RA. Heregulin regulation of Akt/ protein kinase B in breast cancer cells. Biochem Biophys Res Commun 1999;261:897-903.

32 Hung MC, Lau YK. Basic science of HER-2/neu: a review. Semin Oncol 1999;26:51-59.

33 Hellyer NJ, Kim MS, Koland JG. Heregulin-dependent activation of phosphoinositide 3-kinase and Akt via the ErbB2/ErbB3 co-receptor. J Biol Chem 2001;276:42153-42161.

34 Vlahos CJ, Matter WF, Hui KY, et al. A specific inhibitor of phosphatidylinositol 3-kinase, 2-(4-morpholinyl)-8-phenyl-4H-1-benzopyran-4-one (LY294002). J Biol Chem 1994;269:5241-5248.

35 Berrie CP. Phosphoinositide 3-kinase inhibition in cancer treatment. Expert Opin Investig Drugs 2001;10:1085-1098.

36 Vivanco I, Sawyers CL. The phosphatidylinositol 3Kinase AKT pathway in human cancer. Nat Rev Cancer 2002;2:489-501. 